\title{
Controlling a Non-Polynomial Reduced Finite Temperature Action in the U(1) Higgs Model
}

\author{
A. Jakovác ${ }^{1}$, A. Patkós ${ }^{2}$ and P. Petreczky ${ }^{3}$ \\ Department of Atomic Physics \\ Eötvös University, Budapest, Hungary
}

September 21, 2018

\begin{abstract}
An effective theory is constructed for the scalar electrodynamics via 2-loop integration over all non-static fields and the screened electric component of the vector-potential. Non-polynomial terms of the action are preserved and included into the 2-loop calculation of the effective potential of the reduced theory. Also the inclusion of some nonlocal terms is shown to be important. The effect of non-polynomial operators on the symmetry restoring phase transition is quantitatively compared to results from a local, superrenormalisable approximate effective theory.
\end{abstract}

\footnotetext{
${ }^{1}$ e-mail address: jako@hercules.elte.hu

${ }^{2}$ e-mail address: patkos@ludens.elte.hu

${ }^{2}$ e-mail address: ppetrecz@dragon.klte.hu
} 
1. The thermodynamics of high temperature phases and of finite temperature phase transitions of gauge theories is actively studied with help of reduced three-dimensional effective theories [1], 2, 3]. In the construction of the actions of these effective theories substantial progress has been made recently, based on matching the effective theories to the original full finite temperature theory [4, 5]. In this strategy one computes a set of observables in both theories and fixes the relation of the couplings by requiring their agreement. In the high temperature perturbative regime this approach met considerable success [6].

Non-perturbative investigations of full finite temperature theories [7, 8, 9] represent important reference points to every effective model proposition.

Our aim is to investigate a related but conceptually different procedure to arrive at effective models: the partial integration over a set of field variables. This "identity"-transformation should allow to check the accuracy of some physically very appealing candidates for the effective theories of finite temperature phase transitions.

Integration over non-static fields leads to three-dimensional representation of finite temperature field theories. Since the removal of four-dimensional singularities can be fully implemented in this step of partial integration, and in the full theory no intrinsic three-dimensional divergences are present, the emerging theory is finite. This means that any three-dimensional cut-off dependence appearing in the calculation of correlation functions from the reduced theory, will be cancelled exactly by the cut-off dependence of its couplings, induced in the step of partial integration of non-static field variables (PI-step).

In view of the above feature, three-dimensional renormalisability of the reduced model is not required, the presence of higher dimensional or even non-polynomial terms in the reduced action is equally well admissible. The process one follows in the perturbative approach to the reduced theory consists of the following steps:

i) Separation of all couplings of the effective model into a finite and a cut-off dependent part, e.g.

$$
M_{3 D}^{2}\left(\Lambda, T, m_{4 D}^{2}, \ldots\right)=m_{3 D}^{2}\left(T, m_{4 D}^{2}, \ldots\right)+c_{1} \Lambda T+c_{2} \Lambda T \log \frac{\Lambda}{\mu}+c_{3} T^{2} \log \frac{\Lambda}{\mu},
$$

etc. 
ii) Perturbative calculation of physical quantities with the finite parts of the couplings, and using the cut-off dependent part of the action as counterterm.

iii) Separation of the cut-off independent part of the result from the divergent pieces (for a unique separation one has to choose in the course of solving the reduced theory the same scale $\mu$ as introduced above). Since the cancellation of the divergent pieces is exact, the finite part provides the physical answer.

It is interesting to note that in case of the effective potential the threedimensional 1-loop "counterterm" contribution has no finite part, therefore the physical answer (at least up to 2-loop) is simply the finite part of the perturbative contribution calculated with the finite parts of the couplings.

In the present note we shall work out explicitly for the U(1) Higgs model one particularly appealing version of the reduced theory. It arises when also the dynamically screened electric vector-potential component is included into the PI-step. The 2-loop accurate integration will follow the procedure of the gradient expansion. When calculating the local (potential) term of the reduced action we shall find and retain an $\mathcal{O}\left(e^{3}\right)$ non-polynomial contribution. We are able to show that its cut-off dependent part is exactly cancelled when the effective theory is solved.

In the next step the second derivative (kinetic) part of the reduced action is determined by calculating the T-dependent wave function renormalisation of the static scalar and magnetic vector fields, due to the integrated out fields. It turns out that truncating the effect of the screened electric vector component at this stage of the gradient expansion leads to a not fully satisfactory 2-loop effective potential. The formal expression of the 2-loop effective potential calculated directly from the four-dimensional full theory [10, 11] can be reproduced only if a bilocal term of non-locality range $e T$ is taken to represent the effect of the $A_{0}$-integration.

A quantitative comparison will be made between the above complete 2-loop treatment and the approach in which the non-polynomial part of the potential is expanded up to quartic power in the Higgs-field (superrenormalisable approximation). Calculating some data of the first order phase transition restoring the $\mathrm{U}(1)$ symmetry with both approaches, one can assess within the perturbation theory the impact of the higher dimensional operators. 
2. The model is defined with the following Lagrangian density:

$$
\begin{gathered}
L=L_{r e n}+L_{c t}, \\
L_{r e n}=\frac{1}{4} F_{m n} F_{m n}+\frac{1}{2}\left(D_{m} \phi\right)^{*}\left(D_{m} \phi\right)+\frac{1}{2} m^{2} \phi^{*} \phi \\
+\frac{1}{24} \lambda\left(\phi^{*} \phi\right)^{2}+\frac{1}{2} m_{D}^{2} A_{0}^{2}(\mathbf{x}), \\
L_{c t}=\frac{1}{2} \delta Z_{A} F_{m n} F_{m n}+\frac{1}{2} \delta Z_{\phi^{2}}\left(D_{m} \phi\right)^{*}\left(D_{m} \phi\right)+\frac{1}{2}\left(\delta m^{2}+\delta Z_{\phi^{2}} m^{2}\right) \phi^{*} \phi \\
+\frac{1}{24}\left(\delta \lambda+2 \lambda \delta Z_{\phi^{2}}\right)\left(\phi^{*} \phi\right)^{2}-\frac{1}{2} m_{D}^{2} A_{0}^{2}(\mathbf{x}),
\end{gathered}
$$

with

$$
F_{m n}=\partial_{m} A_{n}-\partial_{n} A_{m}, \quad D_{m} \phi=\left(\partial_{m}+i e A_{m}\right) \phi, \quad m, n=1, . ., 4
$$

One notes in (2) the mass term for the static $A_{0}(\mathbf{x})$ field reflecting its Debyescreened nature. All couplings and fields appearing in (2) are renormalised quantities.

The contribution of the non-static fluctuations to the local potential of the static $\phi$-fields can be evaluated up to 2-loops using exactly those diagrams which appear in the direct evaluation of the effective potential [12]. We also use Landau-gauge. The explicit expression is formally the same in terms of two fundamental sum-integrals. The essential difference is the absence of the $n=0$ mode from the sum. As a consequence one can expand these integrals with respect to the mass(es):

$$
\begin{gathered}
\int_{K}^{\prime} \frac{1}{K^{2}+m^{2}} \equiv I^{\prime}(m)=I_{1}+2 I_{2} m^{2}+\ldots \\
\int_{K_{1}}^{\prime} \int_{K_{2}}^{\prime} \int_{K_{3}}^{\prime} \delta\left(K_{1}+K_{2}+K_{3}\right) \frac{1}{K_{1}^{2}+m_{1}^{2}} \frac{1}{K_{2}^{2}+m_{2}^{2}} \frac{1}{K_{3}^{2}+m_{3}^{2}} \\
\equiv H^{\prime}\left(m_{1}, m_{2}, m_{3}\right)=H_{0}+H_{1} \frac{1}{3}\left(m_{1}^{2}+m_{2}^{2}+m_{3}^{2}\right)+\ldots
\end{gathered}
$$

The prime put on the standard notations emphasizes the missing $n=0$ mode. The coefficients of the expansions displayed explicitly have been calculated in 13] with momentum cut-off regularisation, what we also adopt for the present calculation. Since the propagator mass squares of the fields are quadratic in the background field, the non-static contribution to the static potential becomes a polynomial of $\phi^{*} \phi$. The $\operatorname{dim}>4$ terms contribute to the potential starting from $\mathcal{O}\left(e^{6}, e^{4} \lambda, . ., \lambda^{3}\right)$ level, what is negligible relative to the $\mathcal{O}\left(e^{3}\right)$ contribution of the static screened $A_{0}$ field (see below). Therefore we truncate the non-static part at the quartic level. We do not write explicitly out the lengthy expression of the regularised non-static contribution, since we concentrate on handling non-polynomial pieces. 
The static $A_{0}$ integration contributes the following expression to 2-loop accuracy:

$$
\begin{gathered}
\frac{1}{2} T \int_{\mathbf{k}} \ln \left(\mathbf{k}^{2}+M_{A_{0}}^{2}\right)-\frac{1}{2} T \int_{\mathbf{k}} \frac{1}{\mathbf{k}^{2}+M_{A_{0}}^{2}}\left[m_{D}^{2}-e^{2}\left(2 I_{1}+2 I_{2}\left(m_{H}^{2}+m_{G}^{2}\right)+\ldots\right)\right] \\
-2 e^{4} \phi_{0}^{2} T \int_{\mathbf{k}} \int_{Q}^{\prime} \frac{q_{0}^{2}}{Q^{2}\left(Q^{2}+M^{2}\right)\left((K+Q)^{2}+m_{H}^{2}\right)} \frac{1}{\mathbf{k}^{2}+M_{A_{0}}^{2}} \\
-2 e^{2} T \int_{\mathbf{k}} \int_{Q}^{\prime} \frac{q_{0}^{2}}{\left(Q^{2}+m_{H}^{2}\right)\left((K+Q)^{2}+m_{G}^{2}\right)} \frac{1}{\mathbf{k}^{2}+M_{A_{0}}^{2}} .
\end{gathered}
$$

with $K \equiv(0, \mathbf{k}), q_{0}=2 \pi T n_{Q}, m_{H}^{2}=m^{2}+\lambda \Phi^{2} / 2, m_{G}^{2}=m^{2}+\lambda \Phi^{2} / 6, M^{2}=$ $e^{2} \Phi^{2}$ and $M_{A_{0}}^{2}=m_{D}^{2}+e^{2} \Phi^{2}, \Phi$ being the background. The sum-integrals over the 4-momentum $Q$ in the last two integrals are hard, therefore one can expand the corresponding propagators both in the masses and in the soft momentum k. This technique of evaluation has been used in 114, 15 for pure $\mathrm{SU}(\mathrm{N})$ gauge theory. The leading contribution is arrived at by replacing in (5) $K$ by 0 . Adding just these contributions from the last two integrals to the first two terms one finds the non-polynomial part of the potential energy:

$$
-\frac{T}{12 \pi}\left(m_{D}^{2}+e^{2} \Phi^{2}\right)^{3 / 2}+e^{2}\left(\frac{\Lambda T}{2 \pi^{2}}-\frac{T M_{A_{0}}}{4 \pi}\right)\left(-\frac{\Lambda T}{2 \pi^{2}}+\frac{T^{2}}{6}-\frac{1}{2 e^{2}} m_{D}^{2}\right) .
$$

In addition also divergent pieces $\sim \Phi^{2}$ appear. The further contributions in the last two integrals of (5) do not contribute cut-off independent finite terms to the potential energy to $\mathcal{O}\left(e^{4}, e^{2} \lambda, \lambda^{2}\right)$. The polynomial divergent contributions will not be displayed here, since we concentrate on the consistency of the treatment of non-polynomial terms in the Lagrangian.

When in (6) one uses the fact that $m_{D}^{2}=e^{2} T^{2} / 3$, the effect of the $A_{0^{-}}$ integration can be summarised as

$$
U_{\text {nonpol }}(\phi)=-\frac{e^{3} T}{12 \pi}\left(\frac{1}{3} T^{2}+\phi^{*} \phi\right)^{3 / 2}+\frac{e^{3} \Lambda T^{2}}{8 \pi^{3}}\left(\frac{1}{3} T^{2}+\phi^{*} \phi\right)^{1 / 2} .
$$

A more compact form of the renormalised potential emerges if the renormalisation conditions to be applied to the complete expression refer to the $\mathrm{T}$-independent part of the finite $\mathrm{T}$ expression rather than directly to the $\mathrm{T}=0$ expression of the potential:

$$
{\frac{\partial^{2} U(\phi, \mathrm{T}-\text { indep })}{\partial \phi^{2}}}_{\mid \phi=0}=m^{2}, \quad{\frac{\partial^{4} U(\phi, \mathrm{T}-\text { indep })}{\partial \phi^{4}}}_{\mid \phi=0}=\lambda .
$$


This normalisation scheme simplifies the comparison of different approximations to the effective theory. On the other hand the relation of the renomalised mass parameter to some physical scale at $T=0$ becomes more complicated. For instance the expression of the expectation value of the scalar field at $T=0$ calculated with 1-loop accuracy using counterterms derived from (8) reads as

$v^{2}=-\frac{6 m^{2}}{\lambda}\left[1+\frac{\lambda}{8 \pi^{2}}\left\{(1+\log C)\left(1+18 \frac{e^{4}}{\lambda^{2}}\right)+\frac{1}{2} \log \left(-\frac{\mu^{2}}{2 m^{2}}\right)+\frac{9 e^{4}}{\lambda^{2}} \log \left(-\frac{\mu^{2} \lambda}{6 e^{2} m^{2}}\right)\right\}\right]$.

The scale $\mu$ is the renormalisation scale, which below will be chosen to be $\mathrm{T}$, $C=2 \pi \exp \left(-\gamma_{E}\right)$.

The final result is (without giving explicitly the functions $h_{i}\left(e^{2}, \lambda, ..\right)$ below):

$$
\begin{aligned}
L_{3 D}^{p o t}= & \frac{1}{2} m_{T}^{2} \varphi^{*} \varphi+\frac{1}{24} \lambda_{3}\left(\varphi^{*} \varphi\right)^{2}-\frac{e_{3}^{2}}{12 \pi} Q^{3}(\varphi)+\frac{e_{3}^{3} \Lambda}{8 \pi^{3}} Q(\varphi) \\
& +\left(h_{1} \Lambda+h_{2} T \log \left(\frac{\Lambda}{T}\right)+h_{3} \Lambda \log \left(\frac{\Lambda}{T}\right)\right) \varphi^{*} \varphi
\end{aligned}
$$

with $\varphi=\phi / \sqrt{T}$ and

$$
\begin{gathered}
m_{T}^{2}=m^{2}+T^{2}\left\{\frac{1}{12}\left(3 e^{2}+\frac{2}{3} \lambda\right)+\frac{1}{\pi^{2}}\left[e^{4}\left(\frac{1}{16}+\frac{1}{96} \log C+k_{1}\right)\right.\right. \\
\left.\left.+\lambda^{2}\left(\frac{5}{864}+\frac{7}{864} \log C+k_{2}\right)+k_{3} e^{2} \lambda\right]-\frac{1}{16 \pi^{2}} \log \frac{\mu}{T}\left(e^{4}+\frac{5 \lambda^{2}}{54}\right)\right\}, \\
\lambda_{3}=\lambda T, \quad Q(\varphi)=\left(\frac{1}{3} T+\varphi^{*} \varphi\right)^{1 / 2}, \quad e_{3}^{2}=e^{2} T .
\end{gathered}
$$

The regularisation dependent constants $k_{1}, k_{2}, k_{3}$ have the values:

$$
k_{1}=0.473515, \quad k_{2}=0.0190808, \quad k_{3}=-0.0901793 .
$$

The kinetic terms of the static $a_{i}(x)=A_{i}(x) / \sqrt{T}$ and $\varphi(x)$ fields can be found by studying the contribution of the integrated out fields to the corresponding 2-point functions. As it has been shown by 16, 17, for the calculation of the 2-loop effective action one needs only the $\mathcal{O}(e)$ T-dependent 1-loop corrections of the wave function rescaling factor. In accordance with their conclusion our explicit calculation shows that the contribution from non-static modes is $\mathcal{O}\left(e^{2}\right)$ (including the piece necessary in the $4 \mathrm{D}$ renormalisation). Therefore only the static $A_{0}$ integration should be taken into account in $\delta Z_{\phi^{2}}^{T}$. If one terminates the gradient expansion with the usual 
kinetic term one finds:

$$
\begin{gathered}
L_{3 D}^{k i n}=\frac{1}{4} f_{i j} f_{i j}+\frac{1}{2}\left(d_{i} \varphi\right)^{*}\left(d_{i} \varphi\right)+\frac{1}{2} \delta Z_{\phi_{H}}^{T}\left[\left(\partial_{i} \varphi_{H}\right)^{2}+e^{2} a_{i}^{2} \varphi_{H}^{2}\right] \\
\delta Z_{\phi_{H}}^{T}=-\frac{e^{4} \Phi^{2} T}{48 \pi M_{A_{0}}^{3}} \sim \mathcal{O}(e) .
\end{gathered}
$$

This equation shows that only the Higgs part of the complex scalar field receives T-dependent rescaling. The lower case symbols refer to 3D quantities.

Another alternative, which we are going to claim to be the correct procedure, is to retain the full non-local $A_{0}$ contribution to the Higgs 2-point function:

$$
\begin{gathered}
L_{3 D}^{k i n}=\frac{1}{4} f_{i j} f_{i j}+\frac{1}{2}\left(d_{i} \varphi\right)^{*}\left(d_{i} \varphi\right)+\frac{1}{2} \int_{\mathbf{k}} \varphi_{H}(\mathbf{k}) \varphi_{H}(-\mathbf{k})[\mathcal{M}(\mathbf{k})-\mathcal{M}(0)] \\
\mathcal{M}(\mathbf{k})=-2 e^{4} \Phi^{2} T \int_{\mathbf{p}} \int_{\mathbf{q}} \frac{1}{p^{2}+M_{A_{0}}^{2}} \frac{1}{q^{2}+M_{A_{0}}^{2}} \delta(\mathbf{k}+\mathbf{p}+\mathbf{q}) .
\end{gathered}
$$

$(\mathcal{M}(0)$ is subtracted since the corresponding mass-contribution is already contained in (10])). Since $\mathcal{M}$ is already $\mathcal{O}\left(e^{4}\right)$ the terms completing the nonlocal piece to be gauge invariant can be neglected.

The sum of (10) and of either (13) or (14) represents two alternatives of the $3 \mathrm{D}$ reduced model derived from the full theory with 2-loop accuracy.

3. For the 2-loop computation of the effective potential in the reduced model one needs the propagator, and the cubic and quartic vertex parts from the reduced lagrangian, calculated on a constant background $\varphi_{0}$. The formulae will be presented for the non-local case (14). The local approximation will be commented in the discussion part.

$$
\begin{gathered}
L_{3 D}=\frac{1}{4} f_{i j} f_{i j}+\frac{1}{2}\left(\left(\partial_{i} \varphi_{H}\right)^{2}+\left(\partial_{i} \varphi_{G}\right)^{2}+m_{H}^{2} \varphi_{H}^{2}+m_{G}^{2} \varphi_{G}^{2}\right)+\frac{1}{2} e_{3}^{2} \varphi_{0}^{2} a_{i}^{2} \\
+e_{3}^{2} \varphi_{0} \varphi_{H} a_{i}^{2}+i e_{3} a_{i}\left(\varphi_{H} \partial_{i} \varphi_{G}-\varphi_{G} \partial_{i} \varphi_{H}\right)+q_{111} \varphi_{H}^{3}+q_{122} \varphi_{G}^{3} \\
+\frac{1}{2} e_{3}^{2} a_{i}^{2}\left(\varphi_{H}^{2}+\varphi_{G}^{2}\right)+\frac{1}{24}\left(\lambda_{11} \varphi_{H}^{4}+2 \lambda_{12} \varphi_{H}^{2} \varphi_{G}^{2}+\lambda_{22} \varphi_{G}^{4}\right) \\
\quad+\frac{1}{2} \int_{\mathbf{k}} \varphi_{H}(\mathbf{k}) \varphi_{H}(-\mathbf{k}) \mathcal{M}(\mathbf{k})+L_{3 D, c t}
\end{gathered}
$$

with

$$
\begin{gathered}
m_{G}^{2}=m_{T}^{2}+\frac{\lambda_{3}}{6} \varphi_{0}^{2}-\frac{e_{3}^{3}}{4 \pi} Q, \quad m_{H}^{2}=m_{G}^{2}+\frac{\lambda_{3}}{3} \varphi_{0}^{2}, \\
q_{122}=\frac{\lambda_{3}}{6} \varphi_{0}-\frac{e_{3}^{3} \varphi_{0}}{8 \pi} Q^{-1}, \quad q_{111}=q_{122}-\frac{e_{3}^{3} \varphi_{0}^{3}}{24 \pi} Q^{-3} \\
\lambda_{22}=\lambda_{3}-\frac{3 e_{3}^{3}}{4 \pi} Q^{-1}, \quad \lambda_{12}=\lambda_{22}+\frac{3 e_{3}^{3}}{4 \pi} \varphi_{0}^{2} Q^{-3}, \\
\lambda_{11}=\lambda_{12}+\frac{3 e_{3}^{3} \varphi_{0}^{2}}{4 \pi}\left(Q^{-3}-\varphi_{0}^{2} Q^{-5}\right) .
\end{gathered}
$$


The $\mathcal{O}\left(e^{3}\right)$ expression of the effective potential in 3D Landau gauge is the following:

$$
\begin{gathered}
U_{3 D}^{\text {finite }}=\frac{1}{2} m_{T}^{2} \varphi_{0}^{2}+\frac{\lambda_{3}}{24} \varphi_{0}^{4}-\frac{e_{3}^{3}}{12 \pi} Q^{3}-\frac{1}{6 \pi}\left[\left(e_{3}^{2} \varphi_{0}^{2}\right)^{3 / 2}+\frac{1}{2}\left(m_{G}^{3}+m_{H}^{3}\right)\right], \\
U_{3 D}^{\text {div }}=\frac{\Lambda}{2 \pi^{2}}\left[e_{3}^{2} \varphi_{0}^{2}+\frac{1}{2}\left(m_{G}^{2}+m_{H}^{2}\right)\right]+\text { tree }- \text { level "counterterms" }
\end{gathered}
$$

When the above expressions for $m_{G}^{2}, m_{H}^{2}$ are substituted into the divergent part one easily checks the exact cancellation of the polynomial $\left(\sim \varphi_{0}^{2}\right)$ and of the non-polynomial $\left(\sim M_{A_{0}} T\right)$ divergencies. This makes explicit the consistency of the treatment of the reduced theory with non-polynomial potential to $\mathcal{O}\left(e^{3}\right)$ accuracy.

The local 2-loop contributions come from the same set of Feynman diagrams like the one used for the PI-step. This time the two standard integrals are three-dimensional, and were calculated with cut-off regularisation already in 13 :

$$
\begin{gathered}
I_{3}(m)=\frac{\Lambda}{2 \pi^{2}}-\frac{m}{4 \pi} \\
H_{3}\left(m_{1}, m_{2}, m_{3}\right)=\frac{1}{16 \pi^{2}}\left(\log \frac{\Lambda}{\mu}+\log \frac{3 \mu}{m_{1}+m_{2}+m_{3}}+L_{0}\right),
\end{gathered}
$$

$\left(L_{0}=1.0585301-\log 3\right)$. When choosing (as finally we did in the PI-step too) $\mu=T$, the finite part of the 2-loop local effective potential contribution has the following form:

$$
\begin{gathered}
U_{2-\text { loop }}^{\text {loc }}= \\
\frac{e_{3}^{2}}{32 \pi^{2}}\left[\frac{1}{2} m_{H}^{2}+2 M m_{H}+M m_{G}+m_{H} m_{G}-M^{2}-\frac{m_{H}-m_{G}}{M}\left(m_{H}^{2}-m_{G}^{2}\right)\right] \\
+\frac{1}{384 \pi^{2}}\left[3 \lambda_{22} m_{G}^{2}+2 \lambda_{12} m_{H} m_{G}+3 \lambda_{11} m_{H}^{2}\right] \\
\frac{e_{3}^{2}}{16 \pi^{2}}\left[\frac{m_{H}^{4}}{4 M^{2}} \log \left(1-\left(\frac{M}{M+m_{H}}\right)^{2}\right)+\frac{\left(m_{H}^{2}-m_{G}^{2}\right)^{2}}{2 m^{2}} \log \left(1+\frac{M}{m_{H}+m_{G}}\right)\right. \\
\left.+m_{H}^{2} \log \frac{M+m_{H}}{2 M+m_{H}}-\frac{M^{2}}{2} \log \frac{\left(M+m_{H}\right)\left(M+m_{H}+m_{G}\right)^{3}}{\left(2 M+m_{H}\right)^{4}}\right] \\
\frac{e_{3}^{2}}{16 \pi^{2}}\left(m_{H}^{2}+m_{G}^{2}-2 M^{2}\right)\left(L_{0}-\log \frac{M+m_{G}+m_{H}}{3 T}\right)+\frac{q_{122}^{2}}{16 \pi^{2}}\left(L_{0}-\log \frac{2 m_{G}+m_{H}}{3 T}\right) \\
-\frac{3 q_{111}}{16 \pi^{2}}\left(L_{0}-\log \frac{2 m_{H}}{3 T}\right)
\end{gathered}
$$

$\left(M \equiv e_{3} \varphi_{0}\right)$. One adds to this expression the non-local contribution:

$$
U_{2-\text { loop }}^{\text {non-loc }}=-\frac{e_{3}^{4} \varphi_{0}^{2}}{16 \pi^{2}}\left(L_{0}-\log \frac{2 M_{A_{0}}+m_{H}}{3 T}\right) .
$$


If the contribution of the static $A_{0}$ field would be approximated locally (13), the Higgs-mass $m_{H}^{2}$ would receive an extra contribution, destroying the $\mathcal{O}\left(e^{3}\right)$ cancellation of the non-polynomial divergences. The "counterterm" proportional to $\delta Z_{\phi}^{T}$ would also contribute at 1-loop, corresponding to the expansion of (20) in powers of $m_{H} / M_{A_{0}}$. These remarks strongly favor the use of the complete non-local reduced model when arbitrary high spatial momenta $(<\Lambda)$ are allowed.

The exact form of $I_{3}(m)$ does not contain any $\sim \Lambda^{-1}$ part, therefore the cut-off dependent part of the reduced Lagrangian cannot produce at 1-loop any finite piece. Therefore the finite part of the sum of $(\sqrt[17]{17},(\sqrt{19})$ and $(20)$ gives our final result for the 2-loop finite temperature effective potential of the scalar electrodynamics. When compared with [10, 11] one finds complete formal agreement of the terms independent of the regularisation and of the normalisation conditions. But one should not forget that the couplings and masses appearing in it are given by (16), what is different from the solution of the self-consistent Schwinger-Dyson equations. Still the structural agreement represents a satisfactory evidence for the correctness of our reduced partially integrated model and its perturbative treatment described above.

In order to investigate quantitatively the importance or negligibility of the non-polynomial contributions we evaluate the above expression also in a polynomial (superrenormalisable) approximation. It corresponds to expanding in the potential energy of the reduced model the term $\sim M_{A_{0}}^{3}$ in powers of $\varphi^{*} \varphi$ up to the quartic piece and dropping all the higher dimensional operators «1, 18. A small new feature is that now also the cut-off dependent non-polynomial term in (6) is expanded, what modifies the induced counterterm structure. This leads to the following set for the finite parts of the couplings:

$$
\begin{gathered}
m_{G}^{2}=m_{T}^{2}+\frac{\lambda_{3}}{6} \varphi_{0}^{2}-\frac{e_{3}^{3} \tilde{m}_{D}}{4 \pi}, \quad m_{H}^{2}=m_{G}^{2}+\frac{\lambda_{3}}{3} \varphi_{0}^{2}, \\
q_{111}=q_{12}=\frac{\lambda_{3}}{6} \varphi_{0}-\frac{e_{3}^{3} \varphi_{0}}{8 \pi \tilde{m}_{D}}, \quad \lambda_{11}=\lambda_{12}=\lambda_{22}=\lambda_{3}-\frac{3 e_{3}^{3}}{4 \pi \tilde{m}_{D}},
\end{gathered}
$$

$\left(\tilde{m}_{D} \equiv(T / 3)^{1 / 2}\right)$.

In Fig. 1 we present the effective potential in four different approximations at the respective transition temperatures $(e=2 / 3, \lambda=0.3)$. The potential and the field variable are both scaled by appropriate powers of $m_{H 0}^{2}=-2 m^{2}$. The four curves represent the potential from 1-loop and 


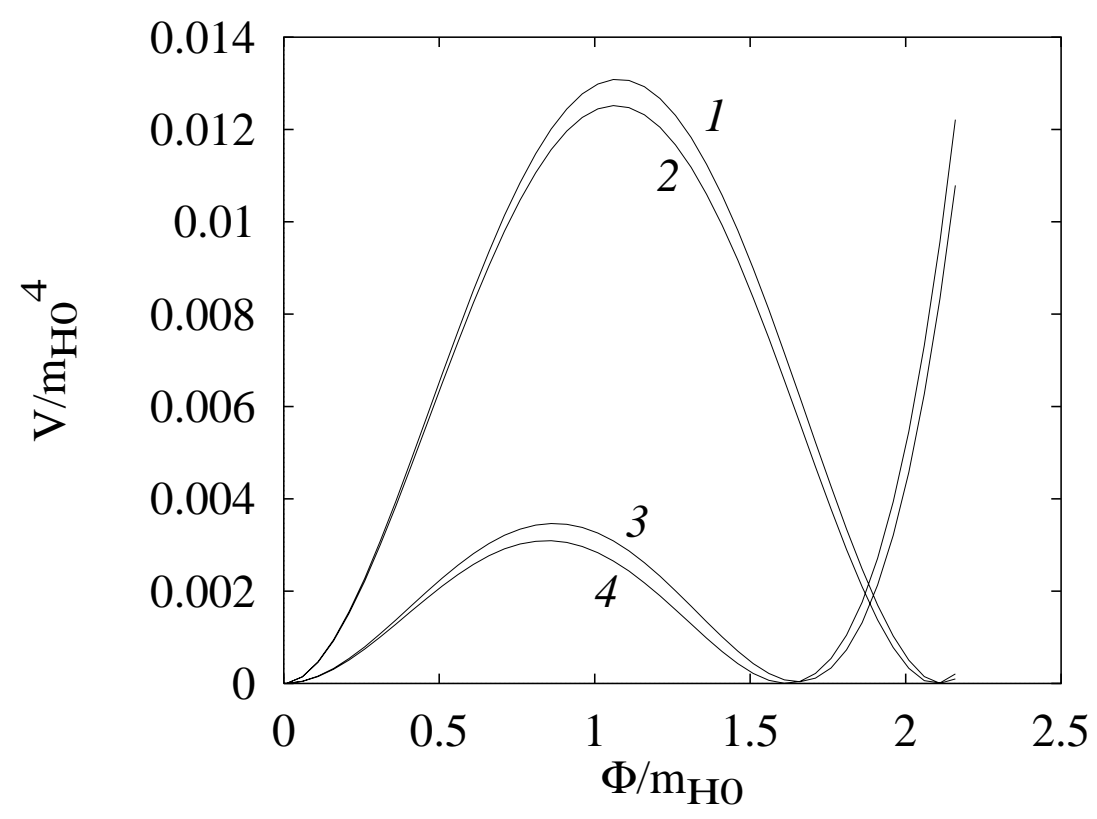

Figure 1: The effective potential at the respective critical temperatures 1. at one loop in polynomial approximation 2. at one loop in nonpolynomial approximation 3. at two loop in polynomial approximation 4. at two loop in nonpolynomial approximation

2-loop approximations computed with superrenormalisable and with nonpolynomial potentials each. The curves show the same qualitative feature as found by [10, 11]. The application of our renormalisation conditions does not change the conclusion that perturbation theory is not reliable for such large value of the coupling $\lambda$. In this specific point $v \approx 1.18\left(-6 m^{2} / \lambda\right)^{1 / 2}$, what leads to quantitative agreement with the results of [10, 11]. The 1-loop correction to $v_{0}$ is fully dominated by the first term of the curly bracket of (9) $\sim e^{4} / \lambda^{2}$.

The effect of the higher dimensional operators is quite noticeable both on the $e^{3 / 2}$ and the $e^{4}$ level. They tend to smoothen the phase transition. Still the result of the simpler superrenormalisable version in this case seems to be quite satisfactory. One might like to characterize the effective potential at the transition with a single number: the interface tension, calculated in thin wall approximation. It is notable that the absolute value of the difference between the polynomial and non-polynomial versions is the same both in 1- 
loop and 2-loop approximations. When measured in units of $\left(-2 m^{2}\right)^{3 / 2}$ one finds:

$$
\begin{aligned}
& \sigma(\text { pol }, 1-l \text { loop })=.227803, \quad \sigma(\text { non }- \text { pol }, 1-l o o p)=.220705 \\
& \sigma(\text { pol }, 2-l o o p)=.090203, \quad \sigma(\text { non }- \text { pol }, 2-l o o p)=.083323
\end{aligned}
$$

The percentual importance of the higher dimensional operators grows to $8 \%$ at two loop, since the surface tension decreases to more than the half of its 1-loop value.

In conclusion it is clear that such analysis will improve our understanding of the physics of the electroweak phase transition too.

Acknowledgements We are grateful for enjoyable discussions with Z. Fodor, I. Montvay and M. Shaposhnikov in the stimulating atmosphere of the CERN Theory Division. Also the grant of the Hung. Science Foundation is gratefully acknowledged.

\section{References}

[1] K. Kajantie, K. Rummukainen and M. Shaposhnikov, Nucl. Phys. B407 (1993) 356

[2] E. Braaten, Phys. Rev. Lett. 74 (1995) 2164

[3] E.-M. Ilgenfritz, J. Kripfganz, H. Perlt and A. Schiller, DESY 95-122

[4] K. Farakos, K.Kajantie, K. Rummukainen and M. Shaposhnikov, Nucl. Phys. B442 (1995) 317

[5] K. Kajantie, M. Laine, K. Rummukainen and M. Shaposhnikov, CERN$\mathrm{TH} / 95-226$

[6] E. Braaten and A. Nieto, Phys. Rev. D51 (1995) 6990

[7] F. Csikor, Z. Fodor, J. Hein, K. Jansen, A. Jaster and I Montvay, Phys. Lett. B334 (1994) 405

[8] Z. Fodor, J. Hein, K. Jansen, A. Jaster and I. Montvay, Nucl. Phys. B439 (1995) 147 
[9] G. Boyd, J. Engels, F. Karsch, E. Laermann, C. Legeland, M. Lütgemeier and B. Petersson, Bielefeld-preprint BI-TP 95/23 (1995 June)

[10] A. Hebecker, Z. Phys. C60 (1993) 271

[11] A. Hebecker, The Electroweak Phase Transition, PhD Thesis, Hamburg, 1995

[12] P. Arnold and O. Espinosa, Phys. Rev. D47 (1993) 3546, E: ibid. 50 (1994) 6662

[13] A. Jakovác, hep-ph/9502313, February 1995

[14] P. Arnold and Chengxing Zhai, Phys. Rev. D50 (1994) 7603

[15] Chengxing Zhai and B. Kastening, hep-ph/9507380, July 1995

[16] D. Bödeker, W. Buchmüller, Z. Fodor and T. Helbig, Nucl. Phys. B423 (1994) 171

[17] Z. Fodor and A. Hebecker, Nucl. Phys. B432 (1995) 127

[18] A. Jakovác, K. Kajantie and A. Patkós, Phys. Rev. D 49 (1994) 6810 